\title{
Research-based quantum instruction: Paradigms and Tutorials
}

\author{
Paul J. Emigh $\odot,{ }^{1, *}$ Elizabeth Gire, ${ }^{1}$ Corinne A. Manogue, ${ }^{1}$ \\ Gina Passante $\oplus^{2}$, and Peter S. Shaffer $\oplus^{3}$ \\ ${ }^{1}$ Department of Physics, Oregon State University, Corvallis, Oregon 97331-6507, USA \\ ${ }^{2}$ Department of Physics, California State University, Fullerton, Fullerton, California 92831, USA \\ ${ }^{3}$ Department of Physics, University of Washington, Seattle, Washington 98195, USA
}

(Received 9 July 2019; accepted 13 December 2019; published 4 December 2020)

\begin{abstract}
A growing body of research-based instructional materials for quantum mechanics has been developed in recent years. Despite a common grounding in the research literature on student ideas about quantum mechanics, there are some major differences between the various sets of instructional materials. In this article, we examine the major instructional considerations that influenced the development of two comprehensive quantum mechanics curricula: Paradigms in Physics (the junior-level physics courses at Oregon State University) and Tutorials in Physics: Quantum Mechanics (a set of supplementary worksheets designed at the University of Washington). The instructional considerations that we consider vary in nature: some are philosophical or theoretical commitments about teaching and learning, while some are practical structures determined in part by the local instructional environments. We then use these instructional considerations as a lens to explore example activities from each curriculum and to highlight prominent differences between them, along with some underlying reasons for those differences. The Paradigms reflect a case where the theoretical commitments drove changes to the practical structures while the Tutorials reflect how theoretical commitments were incorporated into a course with a relatively fixed practical structure. Partially as a result of this large-scale difference, we find that each curriculum prioritizes different theoretical commitments about how to promote student understanding of quantum mechanics. We discuss instances of both alignment and tension between the theoretical commitments of the two curricula and their impact on the instructional materials.
\end{abstract}

DOI: 10.1103/PhysRevPhysEducRes.16.020156

\section{INTRODUCTION}

Quantum mechanics (QM) is an essential part of upperlevel physics instruction. At the undergraduate level, quantum mechanics courses are part of the physics core, forming a foundation for both future coursework and research. Students tend to be excited to study quantum mechanics, which is typically only discussed briefly in high school or introductory physics courses. However, quantum courses can be particularly challenging: they present students with physical behaviors that run counter to students' classical intuitions, and they typically require the use of advanced mathematical techniques.

Over the last 25 years, the physics education research community has accumulated a large body of research on student understanding of quantum mechanics. The research on student ideas about different topics has been particularly

*emighp@oregonstate.edu

Published by the American Physical Society under the terms of the Creative Commons Attribution 4.0 International license. Further distribution of this work must maintain attribution to the author(s) and the published article's title, journal citation, and DOI. broad, including (among others) wave properties of matter [1], probability [2], quantum tunneling [3], time dependence [4-6], measurements [7,8], angular momentum $[9,10]$, and perturbation theory [11]. This research has been supported by results from the development of several formal conceptual assessments [5,12-15]. It has also influenced the development of instructional material aimed at improving student learning of quantum mechanics (see, for example, Refs. [9,16-25]). Each set of material attempts to improve student understanding in different ways and using different pedagogical strategies, many of which have been inherited from the more extensive body of literature on teaching, and more specifically on teaching introductory physics. There has also been research assessing the effectiveness of such curricula (see, for example, Refs. [26-33]).

In this article, we examine two comprehensive sets of instructional material for teaching upper-level quantum mechanics: Paradigms in Physics and Tutorials in Physics: Quantum Mechanics. The first curriculum, Paradigms in Physics $[24,34,35]$, is a reimagined sequence of upper-division courses that makes extensive use of a diverse set of strategies for active student engagement and takes a nontraditional approach to the sequencing of physics content. We focus in this paper primarily on the QM aspects of the 
Paradigms - more detail about some of the nonquantum aspects of the curriculum development may be found in Ref. [36]. The second curriculum, Tutorials in Physics: Quantum Mechanics [23], is a set of supplementary worksheets in the style of Tutorials in Introductory Physics [37] that is intended to support conceptual understanding in a small-group problem-solving setting. Throughout this article, we use the stand-alone terms Paradigms and Tutorials to refer to content that is related to quantum mechanics, and not to refer to the nonquantum Paradigms or to Tutorials in either introductory or other advanced areas of physics.

Each of these two sets of material leverages both the research literature about students' ideas and many years of accumulated pedagogical content knowledge [38,39] in quantum mechanics, though they do so in different ways. In particular, developmental decisions are informed by instructional considerations that are different for the two curricula. We consider two kinds of instructional considerations. Some, which we call theoretical commitments, arise from theories of learning and from instructional philosophies that are more loosely connected to specific theories and to current best practices. We also consider practical structures, which emerge from the institutional and structural environment in which the curriculum is designed and implemented. We acknowledge that these two kinds of considerations are not necessarily distinct, and we have found that they often influence each other. This article represents our collaborative effort to understand these considerations in more detail, and especially to understand how they might lead to differences in the curriculum.

We draw on the authors' experience and knowledge as developers and instructors of the Paradigms (P. J. E., E. G., and C. A. M.) and the Tutorials (P. J. E., G. P., and P. S. S.) to articulate the different theoretical and practical considerations that shaped each curriculum. We also discuss example activities from each curriculum and explore how these activities exemplify the appropriate considerations. We use two curricula to frame this paper in order to draw from a diverse base of theoretical underpinnings and institutional constraints, and we use that base to propose broader conclusions about curriculum development. P. J. E. has been a part of both teams and is in a unique position to be able to compare and contrast the Paradigms and the Tutorials.

We describe example activities from the topic of quantum angular momentum because it forms a rich central point in undergraduate quantum mechanics. Angular momentum is an advanced topic that builds on foundational concepts (like quantum measurements) and it also serves as a core element in analyzing three-dimensional quantum systems such as simple atoms. We hope this paper will thus serve as a useful addition to the published literature focused on the teaching and learning of angular momentum in quantum mechanics $[4,9,10,22,40-43]$.
The main goal of this article is to describe the interactions between theoretical commitments about teaching and learning, practical or structural constraints, and the instructional activities that are developed in these contexts. Section II provides broad overviews of the two curricula. Then, we discuss each in detail: first Paradigms (in Sec. III) and then Tutorials (in Sec. IV). Within each section, we describe that curriculum's theoretical commitments about teaching and learning, the institutional structures in which each curriculum is embedded, and how both these theoretical and practical considerations impact the way activities are written and implemented. In particular, we describe how the theoretical drove the practical in the case of the Paradigms, and how the practical drove the theoretical in the case of the Tutorials. Section V discusses how the variety of theoretical and practical considerations inform each other, help developers make choices, and impact further curriculum development work at the upper-division level. We end in Sec. VI with a message for current and prospective quantum instructors.

\section{BACKGROUND}

Several obvious similarities between the curricula and their development stand out. In particular, the interplay of teaching and research serves as a strong foundation for the designers of each curriculum. Both the Paradigms and the Tutorials have been influenced by the research literature on both teaching and learning and on student understanding of various physics topics. Both curricula make substantial use of active engagement in the classroom, asking students to think about their own thinking and to interact with their peers and with instructors frequently.

The Paradigms and Tutorials classrooms also serve as research laboratories in which both students' ideas and instructional effectiveness have been studied. Although the two research groups have many differences, both emerge from a tradition of social constructivism [44] and share a practical research perspective that the research results should improve the teaching and learning of physics. Both groups also actively incorporate the broader findings of other physics education research.

The research and development groups behind each curriculum operate using an iterative model whereby instructional materials are developed, implemented in the classroom, assessed, and then modified from year to year. Both groups view this iterative model as critical to curriculum development because it blends the results of formal research with practical pedagogical content knowledge of how students interact with particular physics topics and questions.

The substance of the Paradigms and the Tutorials, however, also demonstrate important differences in how they came to exist and in how they are implemented on a day-to-day basis. Below we give a brief overview of the details of what each curriculum is and how it is enacted. 


\section{A. The Paradigms in Physics program}

The Paradigms in Physics program is the set of core upper-division physics courses at Oregon State University (OSU). The centerpiece of the program is a sequence of junior-level courses (each of which is referred to as a Paradigms course). The content of the junior-year courses is structured so that each individual course focuses on a small number of key physical systems and relevant mathematical techniques [16,34]. The courses are modular, meeting every day for $7 \mathrm{~h}$ each week for 5 weeks, including 1 week of integrated mathematical methods content. The classes are pedagogically interactive, making substantial use of a variety of student-centered techniques, including small whiteboard questions [45], small-group problem solving, computer visualization, integrated labs, and kinesthetic activities. These modular, junior-year courses are supplemented with a weekly ( $3 \mathrm{~h}$ ) computational lab and are followed in the senior year by a sequence of more conventional "capstone" courses that synthesize and extend the content from the junior-level courses. Since the beginning of the Paradigms program in 1996, the enrollment has increased from about 20 to about 45 students per year.

The Paradigms began in 1996 as an experimental reimagining of the upper-division physics curriculum at OSU [16]. The design was led by OSU faculty members C. A. M., David McIntyre, and Janet Tate. Since then, the Paradigms has been continuously modified by a combination of the original faculty, new OSU faculty members, postdocs, and graduate students. These modifications have included the development of numerous activities and continuing efforts to resequence the junior-level physics content, including a recent major redesign, Paradigms 2.0 [46].

In this article, we focus on those Paradigms courses that include quantum mechanics content (and specifically, content relevant to angular momentum). McIntyre's textbook, Quantum Mechanics: A Paradigms Approach [47], was developed based on the first several years of the Paradigms program, and is now used as the textbook for all of the quantum courses. The first quantum Paradigms course, Quantum Fundamentals, uses a spins-first approach to introduce the postulates and fundamentals of quantum mechanics, providing students with a simple quantum system that is intended to serve as an analogy for more complicated, future quantum systems. As part of this course, students also begin to learn about position-space wave functions by studying the infinite square well potential.

In the quantum parts of Central Forces (offered toward the end of the junior year), students then explore increasingly more complicated quantum systems culminating in the hydrogen atom. Students learn to apply angular momentum concepts to each of these systems. Throughout the course, students are asked to identify similarities and new features compared to the spin and particle-in-a-box systems studied in Quantum Fundamentals.

In the senior-level Quantum Capstone course, students study advanced quantum systems both by combining previously studied simple systems (e.g., spin-orbit coupling) and by learning and applying more advanced mathematical techniques.

\section{B. Tutorials in Physics: Quantum Mechanics}

Tutorials in Physics: Quantum Mechanics is a set of structured worksheets in the style of Tutorials in Introductory Physics, developed by the Physics Education Group at the University of Washington (UW). The worksheets are intended to supplement lecture instruction in undergraduate quantum mechanics by focusing on conceptual understanding and the building and application of key elements of the quantum model. The worksheets are divided into several sequences that each focus on some aspect of this model. One early sequence introduces students to Dirac notation, function spaces, changes of basis, and finding probabilities $[10,48]$. Another early sequence focuses on quantum measurements and time dependence [32]. The sequence discussed in this article is a pair of tutorials on the topic of angular momentum in quantum mechanics [10].

The Tutorials are given in the junior-level quantum mechanics course at UW. The lecture portion of the course meets for $3 \mathrm{~h}$ each week and has typically used the textbook by Griffiths [49], with most lectures carried out in the traditional format (i.e., relatively little active engagement). Students also meet $1 \mathrm{~h}$ each week in smaller recitation sections, in which the Tutorials are given. The total enrollment of the course has varied from about 50 students to more than 100 students.

Each tutorial includes activities administered after lecture instruction on the relevant topic. Students begin by completing a pretest (typically online) that gives them a first opportunity to express their ideas about the topic. Then, students attend a recitation section where they complete the tutorial worksheet in groups of 3 to 4 , aided by graduate student teaching assistants. After the in-class worksheet, students are assigned 2 to 3 homework problems (in the same style as the in-class questions) intended to let students practice and extend the ideas considered on the worksheet.

The QM Tutorials were initially created during the early 2000s primarily by Andrew Crouse, Bradley Ambrose, Stamatis Vokos, and P. S. S. They were developed at the request of faculty in the department of physics for use in the newly created recitation sections for the upper-level quantum course [4]. Two major periods of development (alongside research on student understanding) followed: the first led by Crouse and P. S. S. (2000-2007) and the second by P. J. E., G. P., P. S. S., and Tong Wan (2011-2018). 


\section{QM PARADIGMS}

In this section we articulate the instructional considerations (both theoretical and practical) of the Paradigms. We begin by describing the theoretical commitments that led to the initial and subsequent development of the Paradigms over the last twenty years. We then identify the practical structures that have also shaped the curriculum. We present the theoretical considerations before the practical ones because one of the defining features of the original design of the Paradigms was to eschew traditional course structures and requirements and mold new structures that fit with the developers' underlying philosophies. Finally, we describe example activities and how they enact the various instructional considerations.

\section{A. Theoretical commitments of the QM Paradigms}

The Paradigms as a whole, including the QM Paradigms, were initially developed by a team of OSU physics faculty including many different individuals. The Paradigms program has continuously evolved since this initial development, an evolution that has resulted in both small and large changes to the curriculum. In this section, we aim to articulate the theoretical commitments that have most influenced the QM Paradigms. An initial list was drafted by E. G., and extensive discussions between P. J. E., E. G., and C. A. M. eventually led to the following five theoretical commitments:

Par-T1 Each individual must make their own set of cognitive connections (individual cognitive connections)

In alignment with Hammer, Elby, Scherr, and Redish [50], we think of people as having mental structures that include interconnected nodes of fine-grained ideas. Since physics concepts, laws, and representations are strongly interconnected, the goal of physics instruction should be to help students develop strongly interconnected knowledge structures about physics. Like Tall and Vinner's [51] construct of a personal concept definition, we recognize that each student comes to a course with their own set of cognitive resources and connections. Instructional activities should be rich enough so that different students can engage with the activities in different ways. Instruction should anticipate that different students will make different connections in any one activity. Therefore, sequences of activities should address a single topic from multiple perspectives or using multiple approaches so that students have many opportunities to make particular connections. A spiral structure should exist in the curriculum so that activities revisit topics or ideas in increasingly sophisticated ways.

Additionally, students should be supported in making connections across different areas of physics. As stated by Manogue et al., "Upper-division students must deal with problems of far greater complexity and must learn to see patterns which cross the boundaries of traditional physics subdivisions" [16].

Par-T2 Social interactions are instrumental to learning and doing physics (social interactions)

Physics ideas are a socially constructed description of the universe. Like Tall and Vinner's idea of formal concept definitions, we recognize that formal definitions of physics topics arise from consensus among the community of physicists [51]. Therefore, a major goal of upper-division physics instruction should be to bring students into the community of physicists and empower them to contribute to the construction of physical descriptions of the Universe. Physics learners should learn to do physics by interacting with their instructors and other physics learners [52]. At this level, instruction should help students begin to identify themselves as members of the physics community. We are influenced by communities of practice in that physics majors should become part of the community of practice around doing physics [53].

Par-T3 Instruction should respond to the ideas that the students in the room have (responsiveness)

Interactions should be a dialogue, with meaningful contributions from both students and instructors. In these interactions, students should participate in professional and productive discussions about physics. We agree with Robertson [54] that classroom instruction should respond and adjust in real time to students' ideas. To do this, instructors should learn about, respect, and value their students' ideas. Students should help by articulating their own ideas and by working to understand the ideas of their peers. Being wrong and refining ideas is a natural part of the process of constructing physics knowledge. Learning environments should facilitate interactions among learners and instructors and be made safe for everyone to participate fully.

Responsive instruction supports students in thinking about their own thinking. Since professionals are metacognitively active, including planning and evaluating their work, students should also engage in these practices. Like Schoenfeld [55], we believe that learning environments should go a step beyond demonstrating the instructor's thinking by providing explicit opportunities for students to make consequential choices when solving problems while the instructional team is present and able to provide support.

Par-T4 Physicists should be representationally fluent (representational fluency)

We are also influenced by ideas from cultural psychology by thinking about external representations (words, diagrams, graphs, pictures, models, etc.) as tools for communicating ideas (shareable objects of thought $[56,57])$. Physical systems and concepts may be externally represented in many ways, and these different representations may communicate different 
aspects of the physics. In order to develop a rich set of cognitive connections, students need to become familiar with the set of representations used by physicists and be able to use these representations flexibly across physics contexts [57-60]. Additionally, students' use of different representations gives instructors more, and more nuanced, information about students' ideas, which provides more opportunities for the instruction to be responsive.

Par-T5 Students must learn how to ask appropriate questions about physical systems (epistemic sophistication)

Learning involves asking and answering questions. These questions arise from seeking connections between ideas, for both personal understanding and to move the community of physicists forward in its understanding. Knowing what kinds of questions are productive to ask about a physical system is an important part of doing physics. The kinds of questions that are meaningful are different for different subdisciplines of physics (e.g., classical vs quantum vs statistical mechanics). Instruction should include explicit discussion of the kinds of questions that are productive for interrogating different physical systems in order to help students develop epistemic competence (i.e., knowing about the nature of physics knowledge and learning physics) [61].

\section{B. Practical considerations of the QM Paradigms}

We now identify several practical structures that grew out of the initial development of the Paradigms:

Par-P1 Class meets every day for 1 or 2 h blocks for a total of $7 \mathrm{~h}$ per week for five weeks (daily schedule)

This schedule is demanding for both instructors and students, but an advantage is that students remember from one day to the next what they were doing. Activities can be long and can bleed over days. To accommodate this schedule, students take fewer courses at a time.

Par-P2 The course instructor leads activities (instructor as leader)

The course instructor typically leads the activities and discussions. They can interrupt an activity with a short clarifying lecture and can easily adjust the sequencing of activities in response to student questions and discussion. Activities may introduce new content or topics; new vocabulary can be introduced immediately to name a concept that students have just "discovered" during an activity. Wrap-up discussions with the whole class provide an opportunity for synthesis. These wrap-up discussions can be productively postponed to the next day as a quick review.

Par-P3 Computers are available to students in class and in study rooms (in-class technology)
Computer visualization is incorporated into classroom activities. Each group of 2 to 3 students is provided a laptop computer (and some students bring their own devices). The instructor's computer can be displayed on monitors around the classroom for demonstrations. A purpose-built simulation of successive Stern-Gerlach measurements is used extensively [62]. Students learn (but may not be proficient with) Mathematica. A study room with computers running the same software is also available to students outside of class. In-class activities with computer visualization can easily be extended to homework.

Par-P4 The course instructor, graduate teaching assistant (TA), and undergraduate learning assistant (LA) are all present at every class meeting (multiple instructors)

The enrollment is growing and currently large enough that the course instructor cannot talk with each group during an activity-in-class TA or LA support is needed. Extensive preclass discussions with the teaching team are a highly valued opportunity to make sure everyone understands the goals and possible student conversations of the activities and to share observations about how the students are doing in order to make adjustments.

\section{Example activities that exemplify the instructional considerations}

Before discussing the examples in detail, we begin by situating the activities within the overall structure of quantum activities in the Paradigms, which are organized around a succession of physical systems that students explore in detail one after the other (each system is shown in a box in Fig. 1). In Quantum Fundamentals, students learn about systems with intrinsic angular momentum (spin-1/2 and spin-1), and are introduced to both the Dirac and matrix representations. At the end of Quantum Fundamentals, we introduce the particle-in-a-box system and the wave function representation. In Central Forces, we introduce three quantum systems: a particle confined to a ring, a particle confined to a sphere (the rigid rotor problem), and the (unperturbed) hydrogen atom [47,63]. These three systems build on each other by introducing one new spatial dimension at a time to help students develop individual cognitive connections (Par-T1). The Ring introduces the $z$ component of angular momentum and the concept of degeneracy. The Sphere introduces $L^{2}$ and the other components of angular momentum. The hydrogen atom introduces all three quantum numbers $n, \ell$, and $m$. Lastly, the Quantum Capstone (a senior-level course) uses the basic quantum building blocks from the junior year to look at quantum systems that are an elaboration on earlier ones using nondegenerate perturbation theory, degenerate perturbation theory, spin-orbit coupling, addition of angular momenta, etc. 


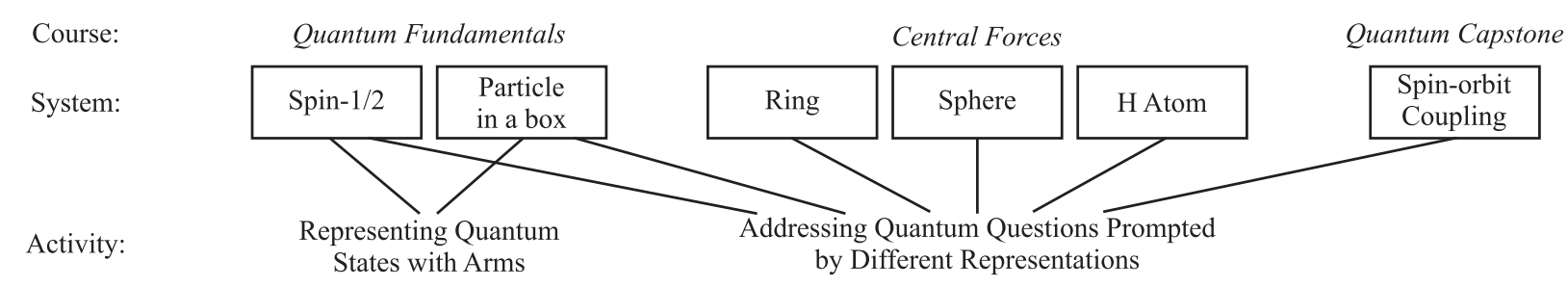

FIG. 1. The sequence of quantum systems considered across the QM Paradigms, with two example activities and where they occur and recur indicated.

Within each of the quantum mechanical systems described above, students participate in a variety of activities, such as solving for eigenstates, exploring the features of different representations, and determining possible measurement outcomes and probabilities. Below, we describe two foundational activities: a kinesthetic activity aimed at representing spin- $1 / 2$ quantum states and a small-group activity focused on multiple representations.

\section{Representing quantum states with Arms}

The QM Paradigms begin with a spins-first approach [47] where students use a computer simulation (Par-P3) of Stern-Gerlach experiments [18] to explore the postulates of quantum mechanics and develop intuitions about quantum measurements. The students learn that the distribution of outcomes for identically prepared particles determines a quantum state [Fig. 2(a)]. Students use the results of Stern-Gerlach experiments to determine Dirac and matrix representations of the states of spin-1/2 particles [ [47], p. 17-25]. During these calculations, students are introduced to the fact that the relative phase between terms determines the state; multiplying a state by an overall phase does not change the state. Note that we denote spin- $1 / 2$ states in Dirac notation using the form $|+\rangle_{y}$ to refer to the state corresponding to "spin up" in the $y$ direction. (a)

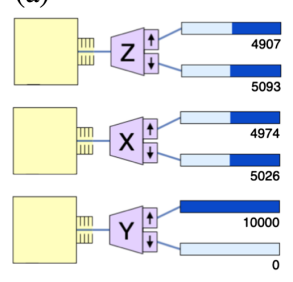

(c) (b)
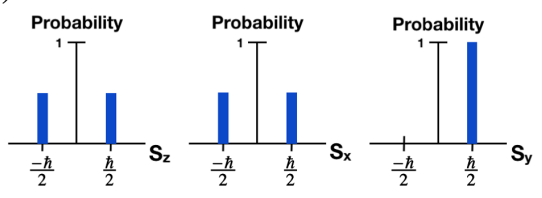

(e)

(d)

$$
1 / \sqrt{2}|+\rangle_{y}+i / \sqrt{2}|-\rangle_{y}
$$

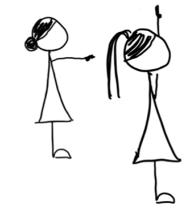

FIG. 2. Various representations of a spin- $1 / 2$ state $\left(|+\rangle_{y}\right)$ : (a) schematic of the results of Stern-Gerlach experiment, (b) histograms of probabilities of values of spin, (c) matrix notation, (d) Dirac (bra-ket) notation, (d) arms notation. Note that $|+\rangle_{y}$ denotes the state corresponding to spin-up in the $y$ direction.
After using diagrams of experiments, histograms of probabilities, matrices, and Dirac notation to represent quantum states [Figs. 2(a)-2(d)], students do a kinesthetic activity [64-67] where they work in pairs to represent spin$1 / 2$ states with their arms. The students stand shoulder to shoulder and use their left arms to sweep out a complex plane: the real axis is forward, parallel to the ground and the imaginary axis points vertically upward [Fig. 2(e)]. The students use their left arms so that, when looking at their own arms, the students see the complex plane in the standard orientation. This activity occurs about 5 instructional hours after the students have done a similar activity where each student represents a single complex number with their arm.

The instructor begins by writing a state such as $|+\rangle_{y}=$ $(1 / \sqrt{2})|+\rangle+(i / \sqrt{2})|-\rangle$ on the board (the state can instead be written in matrix notation). The student standing on the left in each pair is told to represent the probability amplitude (complex coefficient) of the spin-up-in-the- $z$ direction component of the spin state [for this state, $(1 / \sqrt{2})]$. The person on the right represents the probability amplitude (complex coefficient) of the spin-down-in-the- $z$ direction component $(i / \sqrt{2})$. The instructor then says

\section{Instructor: Show me this state.}

For the state $|+\rangle_{y}$, the students could arrange themselves so that the student standing on the left points forward with their arm parallel to the ground and the right students points vertically upward, as in Fig. 2(e) (other arrangements that preserve the relative angle between the students' arms are also correct).

After the students have represented a few states, the instructor then asks

$$
\begin{aligned}
& \text { Instructor: How can you tell the difference between }|+\rangle_{y} \\
& \text { and }|-\rangle_{y} \text { ? }
\end{aligned}
$$

The class then discusses that for $|-\rangle_{y}$, if the student on the left is pointing forward, parallel to the ground, the student on the right should point vertically downward, meaning that it is the angle between the two arms that determines the state.

The instructor then asks

Instructor: Show me $e^{i \pi}|+\rangle_{y}$ ? 
The students could arrange themselves so that the left student points backward, parallel to the ground and the right student points vertically downward. The class then discusses whether or not this state is equivalent to $|-\rangle_{y}$ (it is not-it has a different relative phase).

In this activity, students translate either matrix or Dirac representation (ideally, both) for a spin-1/2 system into arms notation, supporting the development of representational fluency (Par-T4). Although not widely used by physicists (we invented it), "arms" is a pedagogically useful representation $[64,68]$. The students collaborate in pairs to create the arms representations, and students can compare themselves to other pairs' configurations in the room (social interactions-Par-T2). The instructor can see each pair of students and can therefore point out variations and adjust which states the students are asked to represent to accommodate the level of understanding in the room (responsiveness-Par-T3). The prompts are fundamentally open ended, and the fact that quantum states have an arbitrary overall phase means that different pairs of students can make different correct choices and the class can acknowledge these different choices (individual cognitive connections-Par-T5). Similar activities with arms occur later in the course to represent time dependence and then spin precession.

\section{Addressing quantum questions prompted by different representations}

We now describe a touchstone activity sequence entitled, "Angular Momentum and Energy for a Particle on a Ring" [69]. The sequence occurs at the beginning of Central Forces, immediately after lecture content on finding the energy eigenstates for a particle confined to a ring, $\Phi_{m}(\phi)=\sqrt{1 / 2 \pi r_{0}} e^{i m \phi}$, where $r_{0}$ is the radius of the ring and $\phi$ is the azimuthal angle. For this system, the corresponding energy eigenvalues are $E_{m}=m^{2} \hbar^{2} / 2 I$, where $I=M r_{0}^{2}$ is the moment of inertia.

In the sequence, students are given the first two quantum states and asked questions 1-4 in Fig. 3. Students are given the two states one at a time in quick succession (the other two states can be given either in class or on homework as a separate activity). The four states are in fact equivalent but are represented successively in Dirac, matrix, wave function (individuated), and wave function (compact) notations. While the questions for each state are the same, the techniques for answering them differ based on the representation used. Thus, the sequence attends to representational fluency (Par-T4) by asking exactly the same set of questions for the same quantum state but prompted by different representations.

As with most Paradigms activities, the students work together in three-person groups. Social interaction (Par-T2) is promoted by having groups sit at tables with movable chairs around a shared whiteboard, and every student has a marker and can write on this shared brainstorming space.
The Dirac $\left(\Phi_{a}\right)$ and matrix $\left(\Phi_{b}\right)$ notations are computationally the most straightforward since the probability amplitude in each case is the coefficient of each eigenstate. For Q3 in Fig. 3, the fact that one needs to add the probabilities (squared norms of the probability amplitudes) in the case of states with degenerate energies is novel and a precursor to later questions that ask the probability of finding the particle in a particular region of space. For the case of individuated wave functions $\left(\Phi_{c}\right)$, the individual eigenstates are still readily identifiable, but the probability density and the normalization of the eigenstate have been algebraically simplified and students must separate them. The compact wave function notation $\left(\Phi_{d}\right)$ is trickiest. This question can best be posed in homework where students have time to work out the necessary analogue of Fourier series on their own (Par-T1).

Considerable attention is given throughout the QM Paradigms to helping students develop epistemic sophistication (Par-T5). This sophistication is important because it is only possible to ask a few kinds of questions about simple QM systems, and because these questions are quite different in nature from the questions that can be asked about classical systems. For example, most classical mechanics questions are about the position, velocity, or acceleration of a particle, whereas most quantum mechanics questions are about the possible outcomes of a measurement and the corresponding probabilities. To support the development of this sophistication, we first explicitly discuss questions that make sense to ask of classical systems but not in quantum systems. Once we identify productive questions for a quantum system, we then repeatedly ask the same questions with the same wording for many different quantum systems.

Typically only the first two states are considered in class, with the others assigned as homework. The instructor can decide on the fly (Par-P2) which states to consider in class depending on how much help the students need understanding the nuances of the different representations. This feature of the sequence thus demonstrates an important intersection between two of the theoretical commitments: the responsiveness (Par-T3) of the instructor to the ideas in the classroom and the different individual cognitive connections (Par-T1) that students might make both in class and on the homework.

A whole-class discussion addresses the crucial question 5 (see Fig. 3), which further helps students consolidate representational fluency (Par-T4). The whole-class discussion also permits further responsiveness (Par-T3) by allowing the instructor to tailor the exact nature of the discussion to the ideas that the instructional team observed while helping students. Often, student groups may be asked to present their results so that both typical and unexpected solutions are brought forward and discussed.

It is essential to position learning opportunities appropriately to help students make individual cognitive 
In this activity, your group will carry out calculations on each of the following normalized abstract quantum states on a ring:

\begin{tabular}{c|c} 
Dirac notation & $\left|\Phi_{a}\right\rangle=\sqrt{\frac{1}{20}}|3\rangle+\sqrt{\frac{9}{20}}|1\rangle+\sqrt{\frac{9}{20}}|-1\rangle+\sqrt{\frac{1}{20}}|-3\rangle$ \\
\hline Matrix notation & $\left.\mid \begin{array}{c}\vdots \\
0 \\
\sqrt{\frac{1}{20}} \\
0 \\
\sqrt{\frac{9}{20}} \\
0 \\
\sqrt{\frac{9}{20}} \\
0 \\
\sqrt{\frac{1}{20}} \\
0 \\
\vdots\end{array}\right)$ \\
\hline Wave function notation (individuated) & $\Phi_{c}(\phi)=\sqrt{\frac{1}{40 \pi r_{0}}}\left(e^{i 3 \phi}+3 e^{\imath \phi}+3 e^{-i \phi}+e^{-i 3 \phi}\right)$ \\
\hline Wave function notation (compact) & $\Phi_{d}(\phi)=\sqrt{\frac{8}{3 \pi r_{0}}} \cos ^{3} \phi$
\end{tabular}

For each of the following questions, state the postulate of quantum mechanics you use to complete the calculation and show explicitly how you use that postulate to answer the question.

1. For each state above, what is the probability that you would measure the $z$-component of angular momentum to be $-3 \hbar$ ? $0 \hbar ? 2 \hbar$ ?

2. What other possible values for the $z$-component of angular momentum could you have obtained with non-zero probability?

3. For each state, what is the probability that you would measure the energy to be $0 ? \frac{4 \hbar^{2}}{2 I} ? \frac{9 \hbar^{2}}{2 I}$ ?

4. If you measured the energy, what possible values could you have obtained with non-zero probability?

5. How are the calculations you made for the different state representations similar and different from each other? Be prepared to compare and contrast the calculations you made for each of the different representations (ket, matrix, wavefunction).

FIG. 3. The Paradigms activity angular momentum and energy for a particle on a ring. The activity involves four different representations of the given quantum state (above) and asks the same set of questions for each state (below). (Note that $I=M r_{0}^{2}$ is the moment of inertia for the particle on the ring.)

connections (Par-T1). For example, the Ring is similar to the infinite square well potential, which students have studied in Quantum Fundamentals, so the students are not overburdened by extensive new content. However, the Ring has periodic, rather than fixed, boundary conditions, which means that the energy eigenvalues are degenerate. This is the first QM system students encounter that has both a wave function representation and degeneracy. Finally, the Ring is a one-dimensional QM system, so the complications posed by more dimensions and more quantum numbers is not present.

This activity sequence mirrors several other activities given throughout the QM Paradigms. In the preceding Quantum Fundamentals course, students consider very similar activities where they carry out parallel calculations in Dirac and matrix notation for a spin- $1 / 2$ system, and in Dirac, matrix, and wave function notation for the particlein-a-box system. In Central Forces, students will later do the same in the context of two progressively more complicated systems: first for a particle confined to a sphere and second for the hydrogen atom. Lastly, in the Quantum Capstone students consider a system with both spin and orbital angular momentum. The cyclical nature of the activities means that students have additional opportunities to make different individual cognitive connections (Par-T1) in the subsequent activities.

\section{QM TUTORIALS}

We now discuss Tutorials in Physics: Quantum Mechanics in the same fashion as the Paradigms in Physics were discussed. We begin by describing the practical structures that led to the fundamental format of the QM Tutorials. Then, we identify the theoretical commitments that were most important to the efforts to develop specific tutorial activities. Lastly, we highlight a specific sequence of tutorial exercises (for the topic of quantum angular momentum), and detail how the 
development of those exercises was influenced by the instructional considerations (both theoretical and practical). The practical structures are detailed before the theoretical commitments because they placed constraints on the development of the Tutorials. This is in contrast to the Paradigms, where the theoretical commitments more strongly drove curriculum design.

\section{A. Practical structures of the QM Tutorials}

The QM Tutorials were developed over a period of about fifteen years at UW. As described in Sec. II B, the overall style was originally modeled on the introductory Tutorials, which had already been in use for many years. (The introductory Tutorials, in turn, were heavily influenced by the Physics by Inquiry curriculum used primarily to prepare future science teachers [70].) Around the time the QM Tutorials were developed, the upper-division quantum mechanics courses at UW added a fourth credit hour in the form of a recitation, and some faculty in the department expressed a desire to have materials similar to the introductory Tutorials used in these sections. Part of the reason for the similarity between the two types of tutorials is that some of the practical structures that led to the development of the introductory Tutorials (e.g., use in recitation sections as a supplement to large lecture sections) were also true of the quantum mechanics course at UW.

Tut-P1 The QM Tutorials are intended for use in weekly small-group "recitation" sections that may be led by graduate student TAs (recitation sections)

The Tutorials are designed for and administered in recitation sections with $20-25$ students, in contrast to lecture, which can have upwards of 100 students. These recitation sections may be taught by the course instructor or by graduate student TAs at large institutions. In both cases, the instructor may not be familiar with research on student understanding of quantum mechanics. Moreover, TAs may have varying levels of experience with either the material or with implementing active engagement. It is therefore important to have curriculum that has carefully scaffolded activities with carefully worded question prompts. This helps ensure that each group of students is productively engaged while also limiting the effect of different tutorial instructors.

Finally, since the tutorials are held weekly rather than daily, the worksheets are designed to be mostly self-contained. Although students need to draw on and build upon ideas developed in prior tutorials, the contexts for each tutorial are mostly distinct.

Tut-P2 The QM Tutorials are supplementary to lecture instruction (supplementary curriculum)

Instruction in many quantum mechanics courses is primarily through lecture and textbook. Since the tutorials are intended to be a supplementary curriculum, they need to be consistent with the approach and content covered in the textbook (in this case, Griffiths). We therefore developed the tutorials to bolster and expand student understanding of topics already introduced in lecture. They do this by focusing on concepts and reasoning skills that research has identified as being difficult for many students after such instruction.

Tut-P3 The QM Tutorials use limited technology (limited technology)

During development of the Tutorials, it was recognized that they may be used in rooms with limited technology. Therefore, the tasks were designed to be given on paper to students, who complete them collaboratively on a large working space such as a tabletop whiteboard. The findings are then copied onto each student's worksheet.

Tut-P4 The QM Tutorials are intended to be readily adopted by other instructors (adoptable)

The Tutorials were explicitly designed for use beyond the local environment in which they were developed (UW). Thus, care was taken during development to monitor not only how students interpreted the individual questions, but also the extent to which each new group of TAs and instructors was able to identify the goal(s) of a given question sequence. In addition, the results of ongoing assessments of student learning were used to modify the curriculum until the impact was reproducible across different quarters with different instructors.

\section{B. Theoretical commitments of the QM Tutorials}

The QM Tutorials were born out of the practical structures listed above. A major goal during development was to make as big a difference in student understanding as possible in only $50 \mathrm{~min} /$ week. Ongoing research to identify what students could and could not do after lecture instruction was the primary factor that motivated the design and modifications to the curriculum. However, underlying beliefs and models about how students learn and broader goals for the students also played important roles.

It should be noted that the Tutorials include contributions from a variety of researchers at UW, each having shared research and teaching experiences, but also bringing somewhat different perspectives to the development of the curriculum. Below, we summarize five of the shared theoretical commitments that were most influential to the design of the activities discussed in this paper. These were identified by P. J. E., G. P., and P. S. S. reflecting on the curriculum, with additional insights from the development of Tutorials in Introductory Physics and Physics by Inquiry.

Tut-T1 Having a coherent framework is important for reasoning in both familiar and new contexts (coherent framework)

Research and teaching experience suggest that after standard instruction, many students do not develop a 
coherent framework that helps promote successful reasoning, especially when transferring knowledge from one context to another [71]. By framework, we mean a set of physics rules and principles coupled with the criteria for when they apply and the knowledge of how to use them. While the Tutorials use a variety of individual strategies to improve student understanding (many of which target particular difficulties, as described below), the "broader structure of experiments and exercises [is] intended to guide the construction of a coherent conceptual framework" [72]. Particularly common mechanisms used to this end in the Tutorials are to focus student attention on fundamental concepts, encourage the creation of links between concepts, and promote the use of multiple representations.

Tut-T2 Many student responses are predictable, persistent, and transcend context (predictable responses)

We recognize that students bring a broad array of knowledge into the classroom, both from prior courses and from everyday experiences. Research has revealed the existence of certain patterns of answers or chains of reasoning that are prevalent $[71,73]$. When these patterns lead to incorrect answers, they are given the term difficulties. Heron notes that a "difficulty is not the specific idea or reasoning pattern, it is the use, or misuse, thereof" [72]. In many cases, these seem to arise from the broad array of knowledge and realworld experiences that students bring into the classroom. We have found it critical to account for this knowledge during instruction. Many tutorial activities are specifically designed to address particularly persistent difficulties that have been identified through research.

Tut-T3 Understanding is more than just (symbolic) answer-making (nonsymbolic reasoning)

Deep understanding of physics is reflected not solely by an ability to give correct answers, but also by the ability to explain how an answer is determined (reasoning) and to interpret the meaning of an answer (sensemaking) [74,75]. As articulated by Shaffer and McDermott, the goal of a tutorial "is not to deliver additional information but to help students deepen their conceptual understanding and develop skill in scientific reasoning" [76]. Since lecture instruction is often mostly symbolic, the Tutorials frequently ask students to provide or interpret both verbal explanations as well as to translate between various representations in their explanations.

Tut-T4 New knowledge is constructed on existing knowledge and the process is often best facilitated in a social environment (social constructivism)

Driver et al. argue that scientific knowledge is "socially negotiated" and that education should acknowledge this fact [44]. In particular, the developers of tutorials, as Heron notes, "assume that learners construct new knowledge on the basis of their existing knowledge .... Prior knowledge is viewed both as the foundation upon which new knowledge is built, as well as the building material" [72]. It is especially important that this knowledge is not conveyed by the instructor but is instead built by the students in a social environment. Moreover, the ability to communicate ideas and work productively in groups is an essential skill for most professionals and should be explicitly cultivated in educational settings.

Tut-T5 Structured inquiry can be used to facilitate both learning and the ability to reflect on one's own understanding (structured inquiry)

The preface to Tutorials in Introductory Physics states that "it can be difficult for students who are studying physics for the first time to recognize what they do and do not understand and to learn to ask themselves the types of questions necessary to come to a functional understanding of the material" [37]. Student learning, therefore, benefits from a structure that guides students through a sequence of questions that helps them develop a deeper understanding of the material and at the same time helps them learn to ask themselves productive questions. In the Tutorials, this structure typically begins by asking students to commit to an answer so that they become aware of their own thinking and reasoning, followed by subsequent questions that guide students to construct answers using one or more canonical lines of reasoning. Then, students are prompted to reflect on how the various lines of reasoning they have considered align (or not) and to ask themselves how the different ideas that have been expressed can be reconciled.

This instructional sequence is often implemented as the elicit-confront-resolve strategy documented in prior publications [73]. By learning to ask questions, which are modeled both in the text of the worksheets and by the instructors, students are then able to gain not only physics skills, but also the understanding of how to determine when to apply those different skills. This structure also helps ensure that all students grapple with incorrect ideas that they (or their partners) may have.

An additional benefit of this approach is that it brings incorrect student ideas to the attention of the instructor. This serves to address the practical matter (Tut-P2) that tutorial instructors may not be familiar with student thinking. In essence, it allows for pedagogical content knowledge (PCK) to be embedded explicitly into the curriculum [77].

In the tutorial sections, "the instructor is expected to act more like a facilitator of discussion than a dispenser of knowledge" [72]. Part of the "facilitator" role is to allow students to express their own ideas, to 
listen carefully to students when they do so, and to promote discussions that go beyond the provided structure of a given tutorial or question.

\section{Example activity that exemplifies the instructional considerations}

We now present a sequence of exercises from a single tutorial and discuss how they are influenced by the theoretical commitments and practical structures outlined above. The discussion is primarily limited to this single sequence of exercises, but where necessary we also include closely related exercises that precede or follow the chosen example. We emphasize that this example only demonstrates one instance of how the commitments have impacted the curriculum, and that the same commitments have resulted in different decisions about the structure of other tutorial activities.

The angular momentum sequence is composed of two tutorials: Angular momentum in quantum mechanics and Addition of angular momentum (see Fig. 4). The example that we introduce is taken from the middle of the second tutorial in the sequence (circled in Fig. 4). Prior to working on this tutorial, students have had lecture instruction on angular momentum, the hydrogen atom, spin-1/2, and addition of angular momentum. The students have also completed the first tutorial in the sequence, along with several previous tutorials focusing on inner products, time dependence, and measurements.

The primary learning objective for this activity is that students should be able to determine the possible outcomes of a measurement of $\hat{J}^{2}$ (the square of the total angular momentum, $\hat{\vec{J}}=\hat{\vec{L}}+\hat{\vec{S}}$ ) for a quantum state written in terms of the quantum numbers $l, m_{l}, s$, and $m_{s}$ (this is sometimes known as the uncoupled basis). The general answer is that the allowed values of $j$ (the quantum number associated with $J^{2}$ ) range from $|l-s|$ to $l+s$ in integer steps. This answer can be counterintuitive, and students frequently believe that $j=l+s$ is the only possible value [10]. The possible outcomes of a measurement of $\hat{J}^{2}$ are then given by $j(j+1) \hbar^{2}$ for each possible value of $j$. The activity described below leverages students' understanding

Angular momentum in quantum mechanics

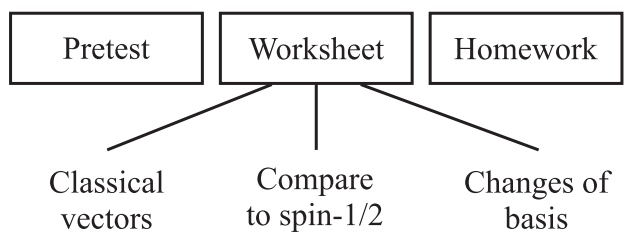

of vector addition in classical physics contexts to build an intuition for why there are multiple possible answers for $j$ and to determine what those answers might be.

The overall structure of the activity (like all the tutorials) is based on structured inquiry (Tut-T5). It uses the elicitconfront-resolve strategy that has been effective in other instructional contexts [73]. Earlier in the tutorial (and on the pretest), students predict whether or not the magnitude of $\vec{J}$ will be well defined (that is, whether or not it has only one possible value). Most students predict (incorrectly) that there is only one possible value, and they tend to pull from a diverse set of resources when answering this and other questions about angular momentum measurements [10]. Since prior research has found that students' framework (Tut-T1) for quantum angular momentum is not always coherent [10], the confront stage of the activity asks students to construct their own answer based on what they know of classical vector addition and quantization. Afterward, they reflect on their original prediction (along with alternate possible predictions).

Below, we discuss the activity itself in two parts: (i) Using classical knowledge to build quantum understanding and (ii) reflecting on possible explanations and resolving inconsistencies. In each part, we describe the exercises given to the students, followed by a discussion of how the exercises highlight the theoretical and/or practical considerations introduced in Sec. IV B. We then discuss two follow-up exercises that reinforce some aspects of the chosen activity.

\section{Using classical knowledge to build quantum understanding}

Throughout the addition of angular momentum tutorial, students consider an electron in the state $\left|l, m_{l} ; s, m_{s}\right\rangle=$ $|2,0 ; 1 / 2,1 / 2\rangle$. Students are first asked to recall relevant knowledge about the angular momentum operators $L$ and $S$ for this state, e.g.,

Determine the magnitude of the orbital angular momentum vector, $\vec{L}$, for this particle. Approximate this value to two decimals in units of $\hbar$. (Hint: It is not just $l \hbar$.)

\section{Addition of angular momentum}

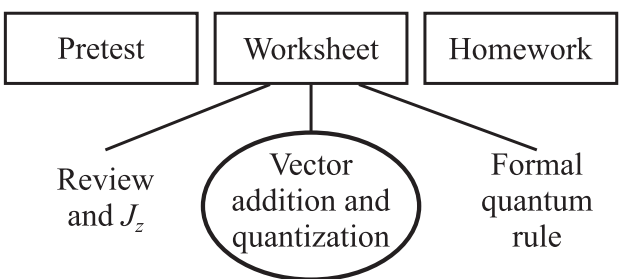

FIG. 4. The sequence of activities associated with the angular momentum tutorials. Each tutorial consists of a pretest, worksheet, and homework assignment, all given after lecture instruction on the corresponding topic. Each worksheet is further divided into three activities - in this article, we describe the vector addition and quantization activity in detail. 
This question is immediately followed by the same question for $\vec{S}$, and students are then asked whether or not the directions of $\vec{L}$ and $\vec{S}$ are well defined.

The core of the activity asks students to use the known quantum values for the magnitudes of $L$ and $S$ $[\sqrt{2(2+1)} \approx 2.45 \hbar$ and $\sqrt{1 / 2(1 / 2+1)} \approx 0.87 \hbar$, respectively], and the lack of certainty about their directions, to construct the classically possible magnitudes of the total angular momentum $(\vec{J}=\vec{L}+\vec{S})$ :

For this sequence of questions, suppose that angular momentum were classical (i.e., that the allowed values for angular momentum were continuous rather than discrete).

1. What is the largest possible value for the magnitude of the total angular momentum vector $\vec{J}$ for this particle? What is the smallest possible value?

2. Draw alignments of the vectors $\vec{L}$ and $\vec{S}$ corresponding to at least three different values for the magnitude of $\vec{J}$.

3. Determine both the largest and the smallest possible values of $J^{2}$ for this particle, assuming that angular momentum can be treated classically. Approximate these values to two decimals in units of $\hbar^{2}$.

The development of this exercise was strongly influenced by social constructivism (Tut-T4). In particular, each group of students constructs the classical behavior of the sum of two vectors whose relative directions are unknown. In early drafts of the tutorial, students were asked to use a graphical version of this argument using cones (see Fig. 5), which is presented in some textbooks [49]. We found that this representation often proved too difficult and misleading for students' first reasoning with a classical argument, and this exercise was moved to the tutorial homework (see Sec. IV C 3). In this instance, the limited technology (Tut-P3) available in the classroom prevented us from using a computer simulation to help students with this

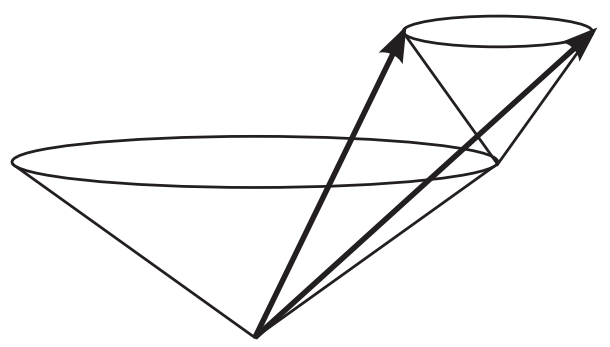

FIG. 5. A graphical representation of the inherent uncertainty in quantum angular momentum. The large cone represents $L$, the small cone $S$, and the two arrows represent two possible (classical) angular momentum vectors with different magnitudes. The cones here are arbitrary (they do not match the state given in the tutorial). visualization, and so we instead chose to develop a task students could complete by hand.

In the next exercise, the students return to the quantum system and make a list of the first four allowed (halfinteger) values of $j$ and the corresponding eigenvalues $j(j+1) \hbar^{2}$. They then compare this list with the classical range they determined previously to build a reasonable set of quantum values for $\widehat{J}^{2}$ for the given system, culminating in the following question:

What are the possible values of $j$ for this electron? What are the corresponding values of $\mathrm{J}^{2}$ ? Explain.

Here, the students are finding an answer for themselves that can be used both to assess their predictions and to account for the fact that the quantum rule gives multiple possible values.

\section{Reflecting on possible explanations and resolving inconsistencies}

The activity concludes with questions to help students reflect on their answers. The first is a student dialogue:

Consider the discussion between two students below. Student 1: "Originally, we knew $l=2$ and $s=1 / 2$. Since $J=L+S$, we just add $l$ and s to get $j$, which would be equal to $5 / 2$ for this particle."

Student 2: "You can't do that because J, L, and S are vectors. Since $L$ and $S$ could point in any direction, the magnitude of $J$ could be any number between the magnitude of $L-S$ and the magnitude of $L+S$, which for this particle would be $1.58 \hbar<J<3.32 \hbar$."

Both students are incorrect. Identify the flaws in each student's reasoning. Explain.

The use of a student dialogue with common incorrect answers is a strategy used throughout the Tutorials. This strategy was primarily chosen to address students' predictable responses (Tut-T2). In particular, student 1's statement highlights the incorrect line of reasoning that our research found was most common in response to questions about $\widehat{J}^{2}$ [10]. Student 2's statement also corresponds to reasoning that is commonly given by students, and is included here to help keep students from overgeneralizing the classical portion of the activity. This dialogue specifies that both statements are incorrect, while in other dialogues, students are asked to agree with one or more of the statements. Because this question specifically asks students to identify how each line of reasoning is incorrect, students must go beyond just providing an answer and instead explore the reasoning underlying the answer. This intersection between the Tutorials' recognition of predictable responses (Tut-T2) and the value of nonsymbolic reasoning (Tut-T3) often leads to particularly powerful learning opportunities for students. 
Student dialogues are included in tutorial activities frequently - in fact, few worksheets do not include at least one student statement or student dialogue. The dialogues exemplify an intersection between the theoretical and practical considerations of the tutorials. In addition to the theoretical commitments mentioned above, student dialogues are a very clear example of structured inquiry (Tut-T5). Practically, the student statements encode pedagogical content knowledge into the text of the activity itself, so that it is easy for TAs in recitation sections (Tut-P1) to reference even if they do not have the relevant prior classroom experience.

The last question in the activity asks students to resolve any inconsistencies between their answers and an earlier exercise in which the students are asked to "predict whether or not the magnitude of the total angular momentum, $\hat{\vec{J}}$, for this electron will be well defined." This question serves as the final step in the elicit-confront-resolve strategy used to structure the overall activity. Explicitly asking students to resolve any inconsistencies is a crucial aspect of structured inquiry (Tut-T5), as students will often proceed without resolving, or sometimes without even noticing, an inconsistency.

At the end of the activity, students are asked to check their answers with an instructor before proceeding (this is very common in the Tutorials). The role of the check out is for students to repeat their explanations verbally and for the instructor to ask probing follow-up questions to get a sense for both their understanding of the coherent framework (Tut-T1) and the sophistication of their nonsymbolic reasoning (Tut-T3). Additionally, the check outs allow TAs to ensure that all students are productively engaged in thinking about the material (Tut-P1).

\section{Understanding in alternate representations}

After the conclusion of the activity above, students work on a third section in which they are reminded of the quantum rule for determining the allowed values of $j$ (covered in lecture prior to the tutorial) and asked to verify, extend, and formalize their findings from the prior section. In their tutorial homework, the students are asked to consider a common textbook representation for angular momentum (the "cone" representation-see Fig. 5) and to describe how this representation might help explain the fact that there is more than one possible value for $J^{2}$. The homework also questions students about the limitations of the cone representation for describing a quantum system (i.e., the angular momentum for a quantum object cannot be represented by a single, well-defined vector).

Both of these follow-up activities ask students to make connections to bolster their understanding of a coherent framework (Tut-T1) for quantum mechanics. Students are asked to revisit the symbolic rule for the allowed values of $j$ in order to link the nonsymbolic reasoning (Tut-T3) from the tutorial with the symbolic answer introduced in class. This is especially important in this case because so many students do not use this rule when making predictions at the beginning of the tutorial, despite the fact that the rule has been previously covered in lecture. Returning to the symbolic rule after an alternate conceptual understanding has been developed is intended to help cement students' ability to use the rule productively in future reasoning. Similarly, considering the same classical argument as in the tutorial using a different representation (the cones in Fig. 5) helps students practice the nonsymbolic reasoning in a new way so that students practice using the reasoning and not just using the rule.

\section{DISCUSSION}

The previous two sections described instructional considerations of two comprehensive quantum mechanics curricula: the Paradigms in Physics and Tutorials in Physics: Quantum Mechanics. We explored both the theoretical commitments of each curriculum and the practical structures within which each curriculum is administered. We then identified the impact of these beliefs and structures on the curricula themselves using example activities to highlight the canonical choices of each set of developers. We now discuss what we have learned from examining the Paradigms and the Tutorials together.

\section{A. Different interplay between theoretical commitments and practical structures}

Both the Paradigms and the Tutorials were influenced by the institutional environment in which they were developed. Despite making distinctions between theoretical commitments and practical structures in the previous two sections, we recognize that they inform each other and a clean distinction between them is somewhat artificial. Additionally, each curriculum has a different relationship to these considerations: in the Paradigms, the theoretical commitments drove changes to the practical structures, whereas in the Tutorials, the practical structures informed the theoretical commitments that were adopted.

The Paradigms were a purposeful redesign of the middleand upper-level physics curriculum intended to center the theoretical commitments, commitments that dictated the practical structures, especially the daily schedule (Par-P1), In-class technology (Par-P3), and multiple instructors (ParP4). The designers of the Paradigms were so committed to the theoretical commitments that they were willing to go to considerable trouble to change the practical structures: establishing consensus among the entire faculty for change; working with the registrar's office to implement a different weekly schedule and course length; and remodeling a classroom for interactive engagement and computer use.

The Tutorials, on the other hand, were designed as a supplementary curriculum (Tut-P2) and given in recitation 
sections (Tut-P1). These constraints were inherited partly from the introductory Tutorials, which were themselves a compromise to bring strategies and methods that had been successful in Physics by Inquiry into the broader undergraduate physics curriculum. But they also arose from departmental circumstances substantially different from those surrounding the development of the Paradigmsnamely, that the number of physics majors at UW was very large at the start of the project and has grown substantially in the subsequent years. Moreover, there was no department-level effort to redesign course sequences taken by majors. Rather, efforts were dedicated to improving student understanding by supplementing lecture instruction in recitation sections using research-based and research-validated materials. For these reasons, the theoretical commitments of the Tutorials are strongly influenced by what can be achieved in the more constrained environment of a weekly 50-min recitation section.

\section{B. Paradigms and tutorials prioritize different theoretical commitments}

In reflecting on the two sets of instructional considerations, we observe both similarities and differences (see Fig. 6). We choose to focus primarily on the theoretical commitments. We note that the theoretical commitments (both for the Paradigms and for the Tutorials) have somewhat different natures. Some are about the nature of knowledge (Par-T1, Par-T5, Tut-T2, and Tut-T4), others are about the nature of learning (Par-T2, Par-T3, Tut-T4, and Tut-T5), and a few are about what is learned or what is important to learn (Par-T4, Par-T5, Tut-T1, and Tut-T3). Many of the commitments share grounding in formal theories about teaching and learning (as identified in Secs. III and IV), but we emphasize that the individually identified commitments (not the underlying theories) most directly influenced the development of each curriculum.

Two unsurprising similarities stand out: both curricula value social constructivism (Par-T2 and Tut-T4) and representational fluency (Par-T4 and Tut-T3). Social constructivism [44] is a theoretical background that has influenced the research groups at both OSU and UW (and many others), and underlies much of the research literature on interactive engagement. Representational fluency is the idea that the ability to understand different representations and to be able to go fluidly back and forth between them is helpful in physics contexts.

Among the remaining theoretical commitments, we articulate three differences in priority between the two curricula.

First, the Tutorials are built primarily to target predictable responses (Tut-T2), where the Paradigms prioritize responsiveness (Par-T3) to attend to students' ideas and to promote individual cognitive connections (Par-T1). In other words, the Tutorials are more structured in an effort to help most students with one or two particularly prevalent
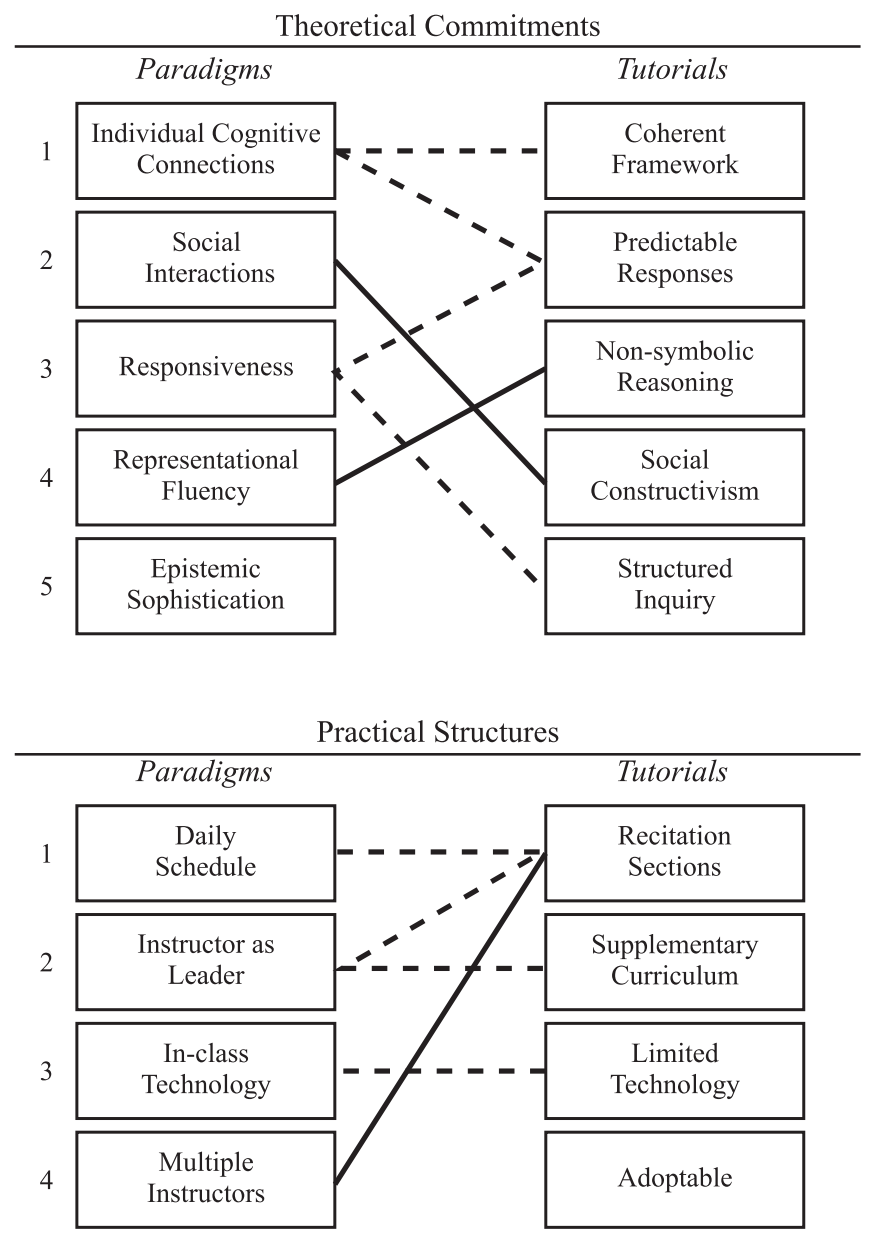

FIG. 6. Instructional considerations for the Paradigms and the Tutorials. The theoretical commitments are listed first, followed by the practical structures. Considerations that we identified as in alignment are connected by solid lines, while considerations that are in tension are connected by dashed lines.

difficulties, while the Paradigms are more agile in an effort to help each student with more individualized concepts.

Each curriculum, however, also acknowledges the theoretical commitment that the other prioritizes. That is, the developers and instructors of the Paradigms are aware of the most common student ideas, and are well prepared to deal with them when they arise. Conversations that have previously helped students come to new understandings in the classroom are well documented in the curricular materials. Similarly, the developers and instructors of the Tutorials know that some students are likely to raise issues that the worksheets are not intended to address. TAs are trained to use Socratic questioning so students can articulate their reasoning and the TA can then respond to each student's needs. However, the TAs at UW are not necessarily experienced instructors and may be unfamiliar with some student ideas. The Tutorials are thus designed to be more targeted and to elicit common incorrect patterns of 
reasoning so they become apparent to the instructors and can be addressed explicitly.

The difference described above leads into a tension between the responsiveness (Par-T5) of the Paradigms and the structured inquiry (Tut-T5) of the Tutorials. The openended nature of the prompts in the Paradigms promotes metacognition by forcing the students to monitor their own reasoning. This metacognition is supported by the responsiveness of both the instructor-student interactions and the whole-class discussions [55]. For example, students are frequently asked to share their (diverse) solutions with the class as a whole, allowing each student to explore a larger set of experiences than is possible for a single group alone. The Tutorials instead use a formal structure in which students are asked to invoke particular knowledge elements in a systematic way intended to help them follow certain productive chains of reasoning in contexts that grow more and more difficult [73]. This kind of structure is followed in almost all of the tutorials, with the long-term goal that students will eventually learn how to ask their own important questions.

A last distinction between the theoretical commitments of the two curricula arises from the Paradigms' commitment to each student building on their own prior knowledge in an order that emerges naturally for them (Par-T1). Multiple opportunities are provided for students to pick up on connections they may have missed. For example, different students might make different connections while working on the Ring activity described in Sec. III, but the additional connections they make in the subsequent Sphere and $\mathrm{H}$ atom activities further each student's knowledge toward a more sophisticated network of ideas. The Tutorials instead aim to have each student build certain knowledge elements and connections at the same point in time, in order to build a coherent framework (Tut-T1), so that those elements can be used for the next activity in the sequence. Opportunities to revisit ideas in increasing levels of sophistication and difficulty must be explicitly built in and not left to the instructors to ensure they occur.

The Paradigms are able to address some issues of equity and inclusion through attending to social interactions (Par-T2) and responsiveness (Par-T3) in the classroom. Norms are established so that when students work in groups, they brainstorm around a big, shared whiteboard and each student has both a pen and an eraser. Students are asked to share ideas not only with their group but also during whole class discussions. The instructor solicits ideas from many different students and takes up students' ideas and language during wrap-up discussions with the whole class. Encouraging participation from all students and positioning students as making valuable contributions can both confirm students' identities as belonging to the community of practice and also challenge students' expectations about what people and what kinds of ideas are valued in physics. These strategies are an explicit topic of conversation among the instructional team. The Tutorials, on the other hand, share only some of these features-for example, students still work on a shared whiteboard and are asked to share their ideas with TAs, but those ideas are not typically shared with the whole classand there is much less explicit attention to or discussion of issues of equity or inclusion in the Tutorials.

The tensions identified above highlight clear differences in the priorities of the designers of the two curricula. However, in each case the Paradigms commitment and the Tutorials commitment are statements about parallel aspects of the same underlying principle. Both curricula, for example, attend to ideas that students have, but the Paradigms attend more directly to ideas definitely present in the classroom while the Tutorials focus more strongly on ideas that research has shown to be especially common. Looking at the full set of commitments for both curricula, all the authors find ourselves in a position where we do not disagree (for the most part) with each other's theoretical commitments, even though we prioritize differently.

\section{Accounting for differences in the curricula with theoretical and practical considerations}

Several obvious differences between the activities in the Paradigms and the Tutorials emerge when we examine the activities discussed in Secs. III and IV. We attempt to account for how these differences arose given the various theoretical and practical considerations.

One difference between the curricula is the specific content (and the amount of content) covered. In this paper, we focus on the topic of angular momentum in quantum mechanics, but we suspect that similar differences are present in other quantum contexts. As a complete curriculum, the QM Paradigms must first introduce and then expand upon angular momentum in quantum mechanics. The overarching structure is spiral, in which students explore angular momentum several times (initially as spin, then as orbital angular momentum through the cycle of Ring, Sphere, and $\mathrm{H}$ atom, and finally combining orbital angular momentum and spin), with each successive instance adding some complexity to the topic while also revisiting the fundamentals introduced previously. In contrast, the Tutorials do not introduce angular momentum, but instead assume that students have previous experience from the lecture class and the textbook. Since the lecture and textbook treatment of angular momentum tends to be highly mathematical (e.g., ladder operators), the Tutorials focus heavily on building conceptual aspects of angular momentum, such as the implications of the fact that all three components of $L$ (or $S$ ) cannot be simultaneously well defined.

Another obvious difference is the style of prompt, which arises primarily out of the tensions between the commitment of the Paradigms to responsiveness (Par-T3) and to students' individual cognitive connections (Par-T1) and 
the structured inquiry (Tut-T5) that the Tutorials use to focus on students' predictable responses (Tut-T2). The Paradigms tend to use a small number of short, open-ended prompts for any single activity. While these prompts may occasionally be written on handouts, they are often written or delivered verbally by the instructor one at a time. The open-ended nature of the prompts give students room to recruit a broad set of prior knowledge and explore and regulate new connections. This prompt structure allows the instructor to respond to students ideas by taking up students' language and changing subsequent questions as warranted. As a result, the curriculum evolves naturally over time in response to formative classroom assessment as well as emerging results of formal research.

Soliciting ideas from all students and responding to them appropriately takes time and involves some risk. Instructors can anticipate common student ideas (documented in the instructor's guide for an activity), or ideas they remember having as students themselves, but students often have ideas that are not anticipated. The instructor may not always know how to respond productively in the moment, which is risky for both the instructor (whose expertise in the room might be threatened) and the student (who might then feel embarrassed about their ideas). Responding to these unanticipated student ideas is helped by having a deep understanding of the physics ideas and a variety of pedagogical moves.

Furthermore, the variety of activity formats in the Paradigms might be difficult for instructors to accommodate and requires a breadth of pedagogical moves. Paradigms activities come in a variety of types (small whiteboard questions, small-group problems, kinesthetic activities, computer visualizations, experiments, etc.). Some activities are very short (5 min or less) and some are long ( $2 \mathrm{~h}$ or more). Being responsive might mean that the order of instructional activities shifts to meet the needs of the students in the room. This high cognitive load on the instructor needed to use Paradigms activities is a potential barrier to implementation. However, the trade-off is creating teachable moments for every member of the class and having timely, meaningful exchanges of ideas.

The Tutorials are composed of worksheets that use a structured inquiry (Tut-T5) approach that guides students to consider particular predetermined lines of reasoning. These lines of reasoning are almost always predictable responses (Tut-T2) that are the result of in-depth research into student ideas about a given topic. The decision to structure the inquiry in this focused way, instead of using a more openended form of inquiry, is in large part due to the practical structures of the Tutorials as a supplementary curriculum (Tut-P2) given in recitation sections (Tut-P1) that have limited time and that are taught by graduate students who do not have years of experience in teaching.

Because the content of the Tutorials (and the training of the TAs) focuses on the most common student ideas, the
Tutorials are not tailored to address a broad variety of student ideas. While the interactions between students and between a group and the TAs does provide a space for students to consider other ideas, inevitably the students are directed back to the questions provided on the tutorial worksheet. This focus means that a tutorial activity might be well suited to helping many students, while not helping a smaller number of students (or helping them only at much greater difficulty for the instructors).

In the Tutorials, all students work in small groups on the activity, which results in slightly different pacing for different groups. Some groups may finish early while others may still be working at the end of class. The potential difference in pacing means that the TAs must be able to assess where a group is in a given activity and to have conversations appropriate to that level. TAs rarely conduct whole-class wrap-up discussions as is common in the Paradigms. However, the trade-off is that it is often possible to have in-depth conversations with individual groups at exactly the most productive time for that group.

\section{Deep similarities: Building ideas, social interactions, multiple representations, and big-picture considerations}

Despite the overt curricular differences discussed above, we also observed some deep similarities between the theoretical commitments and the influence of those commitments on what each curricula tries to accomplish in the classroom. For example, social constructivism underlies at least one theoretical commitment for each group (Par-T2 and Tut-T4). That is, both groups believe that knowledge is constructed by the students and that social interactions are critical to the construction of such knowledge.

Both curricula also value students expressing their knowledge in more than one way: the Paradigms with a very explicit focus on multiple representations and on students translating information between representations, the Tutorials on students articulating the meaning of mathematics and of physical concepts using words and reasoning.

A broader similarity that is not immediately apparent from the examples described here is that the developers of each curriculum take a "big-picture" perspective when designing activities. That is, we each think not only about the local learning goals for a particular activity, but also about how that activity fits into the broader sequence of experiences that we expect students to have over one or more courses. Part of the reason for taking such a bigpicture view can be traced to the practical structures for each curriculum, but there are also strong indicators of the importance of thinking broadly in the theoretical commitments.

Although the Paradigms consists of an entire year-long sequence of junior-level courses, the individual courses are taught by separate instructors and so are not necessarily 
completely coordinated. Over the years, however, the various Paradigms instructors have made an effort (especially in quantum mechanics) to make use of certain activity structures and question types across the different quantum Paradigms. An example of this can be seen in the discussion of the Ring activity in Sec. III C 2, the structure of which is not only repeated throughout Central Forces but in the quantum courses that come before (Quantum Fundamentals) and after (the Quantum Capstone). This structure of repeating the same types of questions about probabilities supports students in making individual cognitive connections (Par-T1) by allowing them to revisit similar reasoning several times over the course of their junior and senior years in progressively more complex contexts.

The Tutorials, which take a more supplementary role, would not necessarily need to maintain cohesive themes across the quantum courses at UW. Each tutorial could focus on addressing student difficulties with one particular context or idea, with little to no coordination between tutorials from week to week. However, the Tutorials commitment to helping students develop a coherent framework (Tut-T1) resulted in the tutorial developers identifying meta goals that span the entire tutorial sequence. Early tutorials (given near the beginning of students' studies of $\mathrm{QM}$ ) tend to focus on helping students identify and implement basic quantum rules, such as the probability postulate, while later tutorials remind students of these rules and help them learn the nuances of using them in more complicated physical scenarios. The example activity discussed in Sec. IV C is primarily an example of the latter, building on students' previously developed intuitions.

\section{MESSAGE FOR INSTRUCTORS}

As demand for research-based instructional materials for the teaching of quantum mechanics increase is increasing, we would now like to address current and prospective instructors directly. The Paradigms and the Tutorials each represent an attempt to leverage research on student understanding, accumulated pedagogical content knowledge, and best practices in education to create activities to help students learn quantum mechanics. The curricula themselves look very different, and are each comprehensive enough that they can look intimidating to prospective adopters. We would like to forefront some of the observations discussed earlier in this paper that may be helpful to instructors who are interested in making use of materials like the Paradigms or the Tutorials but who may not know which to choose or where to start.

First, each curriculum is likely to be particularly easy to implement within a structural environment similar to the one for which it was designed. That is, the Tutorials work well for classes with recitation sections (or similar 50-min chunks of time) that may have large enrollments, while the Paradigms may work better for smaller class sizes and can often be implemented in smaller time chunks. However, we note that each curriculum can be (and has been) adapted for other constraints. For example, the QM Tutorials have been given as interactive tutorial lectures in classes with as many as 150 students. The actual implementation of Paradigms activities can vary substantially from instructor to instructor-they can be implemented flexibly if the instructor is willing to take active steps to ensure pieces continue to fit together as they are changed on the fly, or they can be given following a more proscribed structure.

Second, each curriculum requires preparation beyond just picking up activities and implementing them. The developers of both the Paradigms and the Tutorials have given thought to how an instructor might acquire or develop the skills that instructors at our respective home institutions have experienced personally. The Paradigms relies on daily prep meetings prior to each class, where instructors and TAs work through the same activities that students will, engaging in a dialogue about not only the correct answers but about what students are likely to do and about how to respond to possible student behaviors.

Similarly, the Tutorials require TAs to attend a weekly prep meeting for each worksheet, during which they complete the worksheet while practicing Socratic questioning under the supervision of an instructor or senior TA. Each of these methods of preparation is essential for their respective curriculum because the method of teaching itself can be unfamiliar or challenging to many instructors. Both curricula have instructor guides to help facilitate this preparation [78,79].

Even with this preparation, there are limits and constraints on each mode of instruction. Paradigms activities can become less responsive when class sizes increase. A major feature of Paradigms activities is the instructor taking up students' ideas in front of the whole class. When class sizes increase, it is more difficult for the instructor to be aware of students' ideas (because, for example, more students are talking to a TA) and a smaller fraction of students' ideas are integrated into the instructor's wrap-up discussion for an activity. Students may be more intimidated and less likely to share their ideas in larger classes. On the other hand, the Tutorials are predominantly taught by graduate student TAs rather than by faculty members, and so they are limited by the knowledge of the TAs. Relevant knowledge includes not only content knowledge (how well the TAs know the physics), but also pedagogical content knowledge (how well the TAs know what students think about the physics). Such knowledge can vary substantially from TA to TA, even with adequate preparation.

Lastly, the different theoretical commitments that each curriculum prioritized may help instructors not only choose 
which activities to adopt but also understand aspects of their implementation more clearly. The Paradigms may be especially useful for instructors who value attending directly to their own students' ideas or who emphasize metacognition and self-reflection. In contrast, the Tutorials may be more helpful for instructors who value the construction of a coherent framework for quantum mechanics, or who think their students would benefit from more highly structured materials. Despite these differences in focus, however, both groups share the attitude that teaching with research-based instructional materials should be done thoughtfully: try something out in the classroom, reflect carefully on what happens (and why), and refine it for next year.

\section{ACKNOWLEDGMENTS}

The authors would like to thank the many individuals who contributed to the development of the Paradigms in Physics project (with a special call out to David McIntyre and Janet Tate) and to Tutorials in Physics: Quantum Mechanics (especially, Bradley Ambrose, Andrew Crouse, Paula Heron, Lilian McDermott, Stamatis Vokos, and Tong Wan) over the years. We also thank the instructors and teaching assistants who have made implementing these curricula possible, and the hundreds of students who have learned quantum physics with the help of either the Paradigms or the Tutorials. This paper was supported in part by NSF Grants No. 1323800, No. 1836604, No. 9653250 , No. 0618877, and No. 1022449.
[1] S. Vokos, P. S. Shaffer, L. C. McDermott, and B. Ambrose, Student understanding of the wave nature of matter: Diffraction and interference of particles, Am. J. Phys. 68, S42 (2000).

[2] L. Bao and E.F. Redish, Understanding probabilistic interpretations of physical systems: A prerequisite to learning quantum physics, Am. J. Phys. 70, 210 (2002).

[3] J. Morgan and M. Wittmann, Examining the evolution of student Ideas about quantum tunneling, AIP Conf. Proc. 818, 73 (2006).

[4] A. Crouse, Research on student understanding of quantum mechanics as a guide for improving instruction, Ph.D. Thesis, University of Washington, 2007.

[5] G. Zhu and C. Singh, Surveying students' understanding of quantum mechanics in one spatial dimension, Am. J. Phys. 80, 252 (2012).

[6] P. J. Emigh, G. Passante, and P. S. Shaffer, Student understanding of time dependence in quantum mechanics, Phys. Rev. ST Phys. Educ. Res. 11, 020112 (2015).

[7] G. Zhu and C. Singh, Improving students' understanding of quantum measurement I. Investigation of difficulties, Phys. Rev. ST Phys. Educ. Res. 8, 010117 (2012).

[8] G. Passante, P. J. Emigh, and P. S. Shaffer, Examining student ideas about energy measurements on quantum states across the sophomore, junior, and graduate levels, Phys. Rev. ST Phys. Educ. Res. 11, 020111 (2015).

[9] G. Zhu and C. Singh, Improving student understanding of addition of angular momentum in quantum mechanics, Phys. Rev. ST Phys. Educ. Res. 9, 010101 (2013).

[10] P. J. Emigh, Investigating and improving student understanding of key ideas in quantum mechanics throughout instruction, Ph.D. Thesis, University of Washington, 2015.

[11] G. Passante, P. J. Emigh, and P. S. Shaffer, Investigating student understanding of basic quantum mechanics in the context of time-dependent perturbation theory, in Proceedings of the 2013 Physics Education Research Conference, Portland, OR, edited by P. V. Engelhardt, A. D. Churukian, and D. L. Jones (AIP, New York, 2013).
[12] E. Cataloglu and R. Robinett, Testing the development of student conceptual and visualization understanding in quantum mechanics through the undergraduate career, Am. J. Phys. 70, 238 (2002).

[13] S. Wuttiprom, M. D. Sharma, I. D. Johnston, R. Chitaree, and C. Soankwan, Development and use of a conceptual survey in introductory quantum physics, Int. J. Sci. Educ. 31, 631 (2009).

[14] S. B. McKagan, K. K. Perkins, and C. E. Wieman, Design and validation of the quantum mechanics conceptual survey, Phys. Rev. ST Phys. Educ. Res. 6, 020121 (2010).

[15] H. R. Sadaghiani and S. J. Pollock, Quantum mechanics concept assessment: Development and validation study, Phys. Rev. ST Phys. Educ. Res. 11, 010110 (2015).

[16] C. A. Manogue, P. J. Siemens, J. Tate, K. Browne, M. L. Niess, and A.J. Wolfer, Paradigms in physics: A new upper-division curriculum, Am. J. Phys. 69, 978 (2001).

[17] D. A. Zollman, N. S. Rebello, and K. Hogg, Quantum mechanics for everyone: Hands on activities integrated with technology, Am. J. Phys. 70, 252 (2002).

[18] M. Belloni, W. Christian, and D. Brown, Open source physics curricular material for quantum mechanics, Comput. Sci. Eng. 9, 24 (2007).

[19] S. B. McKagan, K. K. Perkins, M. Dubson, C. Malley, S. Reid, R. LeMaster, and C. E. Wieman, Developing and researching Phet simulations for teaching quantum mechanics, Am. J. Phys. 76, 406 (2008).

[20] C. Singh, Interactive learning tutorials on quantum mechanics, Am. J. Phys. 76, 400 (2008).

[21] A. Kohnle, D. Cassettari, T. J. Edwards, C. Ferguson, A. D. Gillies, C. A. Hooley, N. Korolkova, J. Llama, and B. D. Sinclair, A new multimedia resource for teaching quantum mechanics concepts, Am. J. Phys. 80, 148 (2012).

[22] G. Zhu and C. Singh, Improving students' understanding of quantum mechanics via the Stern-Gerlach experiment, Am. J. Phys. 79, 499 (2011).

[23] L. C. McDermott, P. S. Shaffer, and P. R. Heron (The UW Physics Education Group), Tutorials in Physics: Quantum 
Mechanics, preliminary edition ed. (University of Washington, Seattle, WA, 2014).

[24] C. A. Manogue, P. J. Siemens, J. Tate, K. Browne, M. L. Niess, and A. J. Wolfer, Paradigms in physics: A new upper-division curriculum, Am. J. Phys. 69, 978 (2001).

[25] Adaptable Curricular Exercises for Quantum Mechanics, https://www.physport.org/curricula/ACEQM/.

[26] G. Zhu and C. Singh, Improving students' understanding of quantum measurement II. Development of researchbased learning tools, Phys. Rev. ST Phys. Educ. Res. 8, 010118 (2012).

[27] B. Brown, Developing and assessing research-based tools for teaching quantum mechanics and thermodynamics, Ph.D. Thesis, University of Pittsburgh, 2015.

[28] E. Marshman, Improving the quantum mechanics Content knowledge and pedagogical content knowledge of physics graduate students, Ph.D. Thesis, University of Pittsburgh, 2015.

[29] A. Kohnle and E. Deffebach, Investigating student understanding of quantum entanglement, Proceedings of the 2015 Physics Education Research Conference, College Park, MD (AIP, New York, 2015), pp. 171-174.

[30] A. Kohnle and G. Passante, Characterizing representational learning: A combined simulation and tutorial on perturbation theory, Phys. Rev. Phys. Educ. Res. 13, 020131 (2017).

[31] C. Keebaugh, E. Marshman, and C. Singh, Developing and evaluating an interactive tutorial on degenerate perturbation theory, in Proceedings of the 2016 Physics Education Research Conference, Sacramento, CA (AIP, New York, 2016), pp. 184-187.

[32] P. J. Emigh, G. Passante, and P. S. Shaffer, Developing and assessing tutorials for quantum mechanics: Time dependence and measurements, Phys. Rev. Phys. Educ. Res. 14, 020128 (2018).

[33] G. Passante and A. Kohnle, Enhancing student visual understanding of the time evolution of quantum systems, Phys. Rev. Phys. Educ. Res. 15, 010110 (2019).

[34] C. A. Manogue and K. S. Krane, The Oregon State University paradigms project: Re-envisioning the upper level, Phys. Today 56, 53 (2003).

[35] Webpage of the Paradigms in Physics project, https:// paradigms.oregonstate.edu/.

[36] M. B. Kustusch, C. A. Manogue, and E. Price, From design to secondary implementation: the paradigms in physics ring cycle, Phys. Rev. Phys. Educ. Res. (to be published).

[37] L. C. McDermott and P.S. Shaffer (The UW Physics Education Group), Tutorials in Introductory Physics (Prentice Hall, Boston, MA, 2001).

[38] S. Magnusson, J. Krajcik, and H. Borko, Nature, sources, and development of pedagogical content knowledge for science teaching, in Examining Pedagogical Content Knowledge: The Construct and its Implications for Science Education, edited by J. Gess-Newsomeand and N.G. Lederman (Springer Netherlands, Dordrecht, 1999), pp. 95-132.

[39] H. C. Hill, D. L. Ball, and S. G. Schilling, Unpacking pedagogical content knowledge: Conceptualizing and measuring teachers' topic-specific knowledge of students,
J. Res. Math. Educ. 39, 372 (2008), https://www.jstor.org/ stable/40539304.

[40] D. F. Styer, Common misconceptions regarding quantum mechanics, Am. J. Phys. 64, 31 (1996).

[41] K. S. Taber, Compounding quanta: Probing the frontiers of student understanding of molecular orbitals, Chem. Educ. Res. Pract. Europe 3, 159 (2002).

[42] G. Zhu and C. Singh, Students' understanding of Stern Gerlach Experiment, AIP Conf. Proc. 1179, 309 (2009).

[43] C. Singh and G. Zhu, Students' understanding of the addition of angular momentum, AIP Conf. Proc. 1413, 355 (2012).

[44] R. Driver, H. Asoko, J. Leach, P. Scott, and E. Mortimer, Constructing scientific knowledge in the classroom, Educ. Res. 23, 5 (1994).

[45] Paradigms in Physics: Small Whiteboard Questions, https://paradigms.oregonstate.edu/whitepaper/smallwhiteboard-questions/.

[46] D. Roundy, E. Gire, E. Minot, E. van Zee, and C. A. Manogue, Paradigms in physics 2.0, in Proceedings of the 2017 Physics Education Research Conference, available at https://www.compadre.org/per/perc/2017/Detail .cfm?id=6957.

[47] D. McIntyre, Quantum Mechanics: A Paradigms Approach (Pearson Addison-Wesley, Boston, MA, 2012).

[48] T. Wan, Investigating and improving student understanding of superposition in introductory and quantum physics courses, Ph.D. Thesis, University of Washington, 2018.

[49] D. J. Griffiths, Introduction to Quantum Mechanics, 2nd ed. (Pearson Prentice Hall, Upper Saddle Rive, NJ, 2005).

[50] D. Hammer, A. Elby, R. E. Scherr, and E. F. Redish, Resources, framing, and transfer, in Transfer of Learning from a Modern Multidisciplinary Perspective (Information Age Publishing, Greenwich, CT, 2005), p. 89.

[51] D. O. Tall and S. Vinner, Concept image and concept definition in mathematics with particular reference to limits and continuity, Educ. Stud. Math. 12, 151 (1981).

[52] L.S. Vygotsky, Thought and Language (MIT Press, Cambridge, MA, 1962) (Translated by E. Hanfmann and G. Vakar; originally published in 1934 as Myshlenie i rech').

[53] J. Lave, Situating learning in communities of practice, Perspectives Socially Shared Cognit. 2, 63 (1991).

[54] A. Robertson, Valuing student ideas morally, instrumentally, and intellectually, in Proceedings of the 2015 Physics Education Research Conference, College Park, MD (AIP, New York, 2015), pp. 275-278.

[55] A. H. Schoenfeld, Learning to think mathematically: Problem solving, metacognition, and sense making in mathematics, J. Educ. 196, 1 (2016).

[56] M. Cole and J. V. Wertsch, Beyond the individual-social antinomy in discussions of Piaget and Vygotsky, Hum. Dev. 39, 250 (1996).

[57] D. Kirsh, Thinking with external representations, AI \& SOCIETY 25, 441 (2010).

[58] E. Gire and E. Price, Structural features of algebraic quantum notations, Phys. Rev. ST Phys. Educ. Res. 11, 020109 (2015). 
[59] A. V. Heuvelen, Learning to think like a physicist: A review of research-based instructional strategies, Am. J. Phys. 59, 891 (1991).

[60] E. Hutchins, Cognition in the Wild (MIT Press, Cambridge, MA, 1995).

[61] D. Hammer and A. Elby, Tapping epistemological resources for learning physics, J. Learn. Sci. 12, 53 (2003).

[62] Spins Simulation, Quantum Mechanics, Open source physics qm spin program, https://www.compadre.org/ osp/items/detail.cfm?ID=7011 (2003-2018).

[63] A. Goswami, Quantum Mechanics (Waveland Press, Dubuque, IA, 2003).

[64] C. A. Manogue, E. Gire, and D. J. Roundy, Tangible metaphors, in Prooceedings of the 2013 Physics Education Research Conference, Portland, OR, edited by P. V. Engelhardt, A. D. Churukian, and D. L. Jones (AIP, New York, 2013), pp. 27-30.

[65] A. R. Daane, L. Wells, and R. E. Scherr, Energy theater, Phys. Teach. 52, 291 (2014).

[66] S. M. Grant, The kinesthetic approach to teaching: Building a foundation for learning, J. Learn. Disabil. 18, 455 (1985).

[67] Paradigms in Physics: Kinesthetic Activities, https:// paradigms.oregonstate.edu/whitepaper/kinestheticactivities/.

[68] E. Price, E. Gire, T. Dray, C. A. Manogue, and C. DeLeone, Characteristics of external representations and implications for physics instruction, Phys. Rev. Phys. Educ. Res. (to be published).

[69] Paradigms in Physics: QM Ring sequence, https:// paradigms.oregonstate.edu/sequences/6/.
[70] L. C. McDermott and P. S. Shaffer (the UW Physics Education Group), Physics by Inquiry (Wiley, New York, 1996).

[71] L. C. McDermott and P. S. Shaffer, Research as a guide for curriculum development: An example from introductory electricity. Part I: Investigation of student understanding, Am. J. Phys. 60, 994 (1992).

[72] P. Heron, Identifying and addressing difficulties: Reflections on the empirical and theoretical basis of an influential approach to improving physics education, in Getting Started in PER, 4th ed., Vol. 2 (2018).

[73] L. C. McDermott, Oersted medal lecture 2001: "physics education research - the key to student learning", Am. J. Phys. 69, 1127 (2001).

[74] P. Heller, R. Keith, and S. Anderson, Teaching problem solving through cooperative grouping. part 1: Group versus individual problem solving, Am. J. Phys. 60, 627 (1992).

[75] E. Kuo, M. M. Hull, A. Gupta, and A. Elby, How students blend conceptual and formal mathematical reasoning in solving physics problems, Sci. Educ. 97, 32 (2012).

[76] P. S. Shaffer and L. C. McDermott, Research as a guide for curriculum development: An example from introductory electricity. Part II: Design of instructional strategies, Am. J. Phys. 60, 1003 (1992).

[77] L. S. Shulman, Those who understand: Knowledge growth in teaching, Educ. Res. 15, 4 (1986).

[78] Paradigms in Physics-Instructor Guides, https:// paradigms.oregonstate.edu/ (2019).

[79] Tutorials in Physics: Quantum Mechanics-Instructor Guides, https://sites.google.com/uw.edu/uwpeg/curricula/ tutorials-in-physics-quantum-mechanics?authuser=0 (2019). 